\title{
Design and simulation of microfluidic smart bandage using Comsol Multiphysics
}

\author{
${ }^{1}$ Priti Rajput, ${ }^{2}$ Parveen Kumar Lehana \\ ${ }^{12}$ Department of Electronics, University of Jammu, Jammu and Kashmir \\ 1. pritijamwal@outlook.com, ${ }^{2}$ pklehana@gmail.com
}

Article History: Received: 11 January 2021; Accepted: 27 February 2021; Published online: 5 April 2021

\begin{abstract}
Microfluidics is an emerging field finding its applications in biomedical engineering for investigations of cellular micro structures. Human body is composed of $70 \%$ of water having thin and fine structure of microfluidic blood channels spread throughout the body. These microchannels supply essential nutrients to each part of the body at right time and in right amount. Microfluidics is the science of controlling and manipulating the fluid in micro channels. Manipulating the flow through the microchannels is useful for developing electronic devices, artificial human body parts, and economical diagnostics tools. Microfluidics also helps in manufacturing of pharmaceuticals and carrying out precise chemical analysis of complex systems. A number of diagnostic devices and artificial human organs like lungs, heart, kidneys, etc. have been simulated using microfluidics for developing easy, economical, non-invasive, and rapid method of drug testing. Recently, its applications have also been investigated in smart bandage design. Further, blend of microfluidics with herbal medicines is expected to enhance the healing along with negligible side effects unlike allopathic treatments. The scope of the present research is to develop a smart bandage capable of sensing the status of the wound and supplying required amount of drugs using microfluidic channels. The flow rate of drugs through microchannels is simulated using the physics of laminar flow, capillary action, and diffusion phenomena for optimizing the size and shape of the constituent components of the bandage like microfluidic channels, mixers, and porous material used for drug distribution with in the active area of the bandage. The analysis of the results shows that the mixer having inner radius as 150 microns and outer radius as 250 microns is sufficient to mix the incoming drugs via inlets of 50 microns' diameter. Results also show that capillary action dominates the diffusion phenomenon for supplying the drugs to the wound. The investigations of the prototype show that a smart bandage having the provisions of uniform drug distribution, automatic control, on board $\mathrm{pH}$, moisture, $\mathrm{O} 2$ measurement, and dc current based healing mechanism is possible to be incorporated with in a comfortable size for fast wound healing.
\end{abstract}

Keywords: microfluidics, microchannels, smart bandage, micro drug mixers, automatic channel flow.

\section{Introduction}

Life came into existence on this planet billion of year ago and till date evolution is going on. The species are evolving and technological advancements are bringing new reforms to the life every day. With the advancements in technology, the demand for smart environment is pacing up and the researchers worldwide are contributing towards it [1]. The day is not far when the researchers will be able to mimic human brain on a microfluidic chip. Microfluidics is the study of controlled flow of fluids through microchannels. Microfluidics originated 20 years back and it is widely used by researcher's worldwide now. In microfluidics, fluid is the main constituent flowing through capillaries in micro or pico litres per unit of time [2]. Finite control of the ingredients, least wastage, small sample size, precise control of fluids, and real time monitoring are the main advantages of microfluidic systems [3]. A microfluidic robotic chemist, can help in the development of new compounds because of its precise control capabilities [4]. Microfluidics is also helping in the development of economical and environment friendly wearable devices [5] [6]. Paper microfluidic devices are gaining popularity because of being light in weight and disposable in nature [7]. A number of diagnostic devices are being fabricated using the principles of microfluidics [8] [9]. Artificial lungs with biomimetic branches, microfluidic channels, and gas transfer membrane have also been reported [10] [11]. The microfluidic drug delivery systems having the capability of supplying the drugs to a specific small volume of the body have also been investigated [12]. Effect of medicines on human organs has been investigated using artificial lungs, livers, and hearts. Microfluidics devices provides an easy, economical, non-invasive, and rapid method of testing drugs [13]. Nanoparticle drug affinity is used to carry the drug in required quantity to the infected site. On contrary, conventional system carries the drugs to those parts of the body also that do not require the dose and results in ill effects [14]. Microfluidic and nanofluidic methods limit the supply of drugs to the specified tissues only. Beside healing of the internal tissues, sometime a simple wound may result in chronic wound if proper treatment is not given in time. It is interesting to note that Ayurveda has a lot of herbal medicines, generally each one having almost negligible side effect. In ancient India and China, Ayurvedic system of medicines was very prevalent and effective [15]. Although allopathic medicines have dominated the Ayurvedic practices, but with the awareness, the demand of Ayurvedic medicines is increasing. Sometimes the small dose of antibodies used to control the 
virus (or bacteria) results in adaptation of the virus (or bacteria), leading to the need of higher doses of the same antibodies. It has been reported that allopathic system renders the body weak and hence, time demands a shift from allopathic to ayurvedic medicinal system [16]. Turmeric has been reported as antibacterial and antioxidant for natural healing the wounds [17] [18]. Herbal extracts promote blood clotting and helps in fighting infection by its antibacterial properties. The natural agents i.e. herbal extracts boost the regeneration of cells [17]-[19]. Honey is another important ingredient used in wound healing. It has antioxidant, antibacterial, and scavenging properties [20] [21]. Gelam honey is also a good antioxidant. Low pH of honey does not allow the bacteria to survive so long as the honey is not diluted [21]. Garlic can also be used to reduce the rate of spread of infection due to allicin present in it [22]-[25]. Glutamine present in spinach helps in increasing collagen synthesis resulting in fast wound healing. [26] [27]. Nimbidin in neem is rich in sulphur giving antifungal and antibacterial capabilities to it [28]. Tulsi has been reported to pace the healing due to anti-oxidant and anti-inflammatory property [29]-[30]. Coconut oil has also been reported as beneficial in wound healing [31].

Skin is a natural biosensor for the sensation of touch, heat, and cold along with regulating the body temperature. It protects from harsh environment and keeps safe from disease causing microbes. Skin consists of three layers' epidermis, dermis, and hypodermis. The outermost layer is epidermis and acts as a water proof barrier. New cells grow at the bottom of epidermis and travel to the top till wearing off. The dermis is present below the epidermis containing sweat glands, hair follicles, and connective hard tissues. The deepest layer is called hypodermis. It connects the skin to the bones and muscles. It provides skin with nerves and blood supply. It is made up of fat that restores the elasticity of the skin. It protects from pathogens, controls water evaporation, and prevents nutrients from being washed by [32]-[34]. Automated healing mechanism of living beings comes into action even for a small cut on the skin. The automated mechanism is activated whenever some of the parameters like $\mathrm{pH}, \mathrm{O}_{2}$, glucose etc. change. On the start of the healing, the $\mathrm{pH}$ increases during granulation and decreases afterwards to settle around the value of 4-6. Impaired healing results in oscillatory $\mathrm{pH}$ between 7-8. Cell proliferation, antibacterial activities, and blood vessels growth are controlled by the presence of $\mathrm{O}_{2}$. It has been reported that infected wounds have glucose concentration around 0.3 to $1.0 \mathrm{mM}$ instead of $5.0-7.6 \mathrm{mM}$ for noninfected ones. Biomarkers are widely used for estimating the level of glucose in the vicinity of the wound [35] [40].

There are four stages of wound healing, haemostasis, inflammatory, proliferative, and maturation. The first phase of healing is haemostasis. A cut or broken blood vessel shrinks immediately and slows down the blood flow. There are tiny proteins called clotting factors in the blood that play major role in restricting the blood flow [35] [36]. During haemostasis, the platelets change shape and release chemicals attracting more activated platelet towards it. Platelets stick to each other and form a platelet plug. In case of a torn tissue, information is sent to the clotting factors floating in the nearby blood and fibrin is produced that in turn tightly bounds the platelets leading to coagulation [37] [38]. In inflammatory stage, vascular inflammatory response increases the blood flow to the deficient area. Thrombin helps in transporting inflammatory cells to the injury site by increasing vascular permeability. Blood vessels dilate to allow essential cells, antibodies, white blood cells, growth factors, enzymes, nutrients to arrive at the wound site for healing [39]. In proliferation stage of healing, healthy granulation is initiated due to fibroblast receiving sufficient $\mathrm{O}_{2}$ and nutrients from the blood stream [35] [37] [40]. The colour of the granulation tissue indicates the healing status of the wound. Dark granulation means infection and poor perfusion. Maturation is the last stage of healing and it may last for a year. Unnecessary vessels formed in granulation are removed by apoptosis replacing Type III collagen by Type I collagen [35][40].

The process of natural healing can be enhanced by using external medicines and protection from microbes damaging the wound. It is clear from the literature survey that the wound can be healed much faster if $\mathrm{O}_{2}$, heat, antibacterial drugs especially herbal, moisture, and $\mathrm{pH}$ level are controlled precisely. The scope of present research is to develop a smart bandage capable of sensing status of wound and supplying required amount of medicines using microfluidic channels. The results of simulations of the flow of medicines through the proposed structure of the bandage are presented in this paper. Comsol Multiphysics is used for simulation of the flow through microfluidic channels. The status of the research carried out in the domain of smart bandages is presented in the following section. The detail of the proposed smart bandage and the methodology of the investigations carried out is given in Section II. Section III presents the results and discussions. Conclusions and future scope are presented in Section IV.

\section{Worldwide progress in bandage design}

A conventional bandage comprises of a simple medicated strip in central part of the bandage. Liquid bandages are also commonly available which can directly be sprayed on the wound. It relieves the pain by covering nerve endings. It controls the moisture and safeguard against bacteria, debris, and environmental hazards. Sumithra et al [41] in 2016 reported a neem based herbal bandage. The structural part consisted of cellulose and cotton. The neem leaves were dried in shadow and converted into powder extract. The powder was dissolved in a solution of methanol for a night keeping in shaking mode. The extract was filtered by Whatman (No 1) paper and liquid 
allowed to evaporate at room temperature. The filtrate was tested on the subjects for antifungal and antibacterial properties. Cotton fibres must be sterilized to avoid bacterial growth before using it for making bandages [42]. Hardy [43] et al reported a bandage for enhancing the blood clotting rate using a mesh comprising of alternate gold strings for supplying voltage to the wound site (Fig. 1). The current passing through the wound doubles the clotting rate without any damage to the surrounding tissue.

Tamayol [44] et al reported a microfluidic bandage equipped with onsite heaters. The heaters start operating on encountering a bacterial infection and the drug is automatically released by the action of heat stimuli. Amjadi [45] et al reviewed recent techniques of smart bandage design and characterized them in four major groups depending upon the method of triggering mechanism namely electric, heat, light, mechanical, and psychological stimuli. Pan [46] et al reported a color changing fibrous material

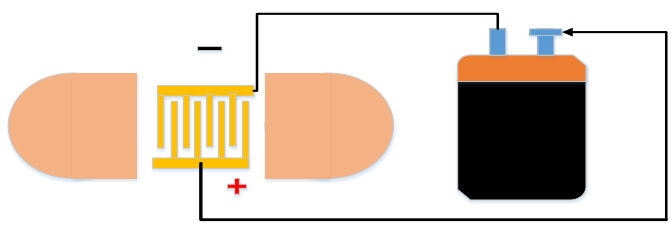

Fig. 1 Design of gold plated smart microfluidic bandage system for speeding hemostasis [43]. for real time monitoring of wound $\mathrm{pH}$. The change in colour is an indication of $\mathrm{pH}$ change and it monitors the healing process without the need of visiting a doctor frequently. It is reported in the research that wounds can also be monitored through wireless technology [47]. Similar efforts for colour based detection has been used at Fraunhofer Research Institution for modular solid state technologies [48]. The researchers at University of Bath and University of Bristol reported a prototype of bandage for detecting bacterial colonies based on nontoxic florescent dye that turns green on encountering bacteria. Efforts have also been done for on-board sensing of $\mathrm{pH}$, metabolites, enzymes, $\mathrm{O}_{2}$, etc., by benzalkonium chloride and pyranine based sensing [49] [50]. Mostafalu [51] et al experimented with thread based encapsulation of drugs with independent addressing to release the medication (Fig. 2). The signal is sent wirelessly to the microcontroller and a voltage signal is sent to a specified fiber to heat the gel enriched in infection fighting antibiotics. On heating, the gel is liquefied and goes to the wound.

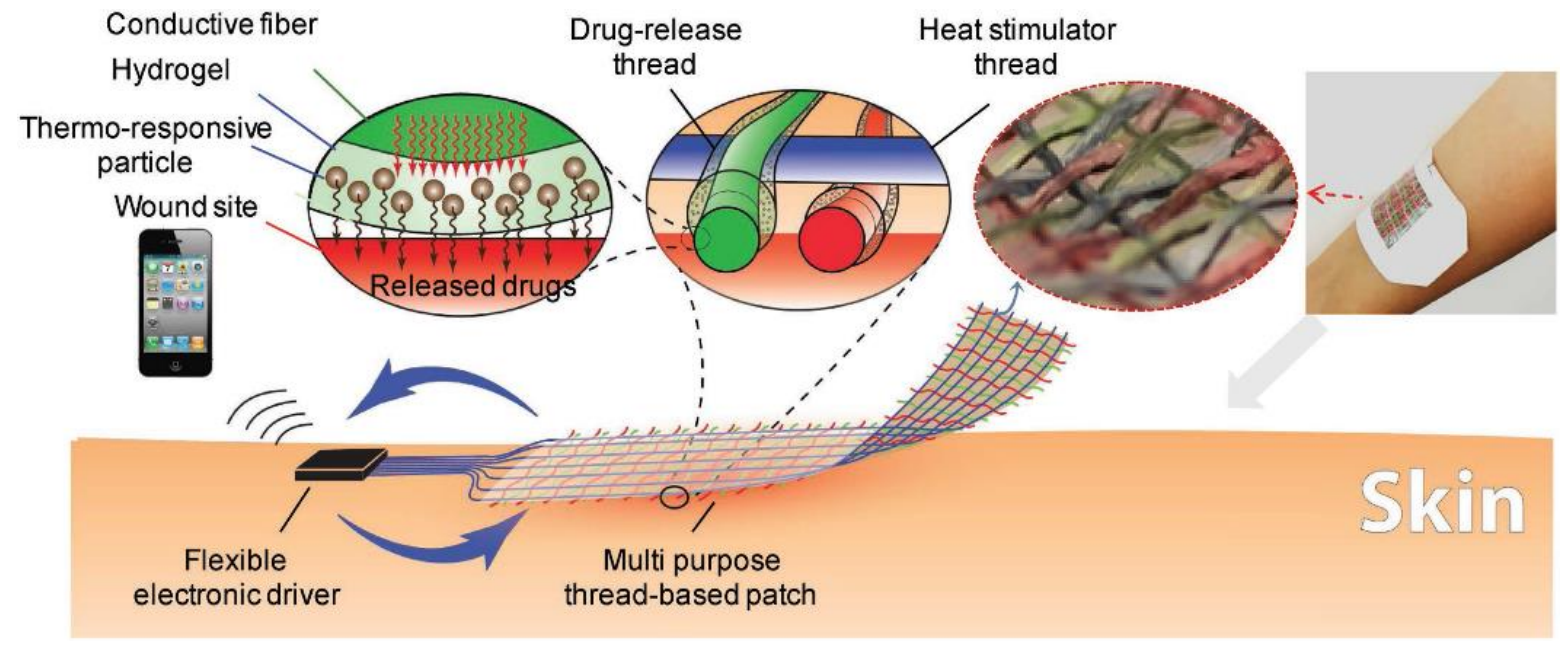

Fig. 2 A smart bandage fabricated with heaters that initiates drug release on the receipt of signal [51].

Sayed [52] et al reported a biological bandage comprising of animal collagen, progenitor cells, and dendrimers to speed up the process of healing. Dendrimers migrate and eliminates the bacteria on encountering the infection. Farooqui [53] [54] et al reported a low cost smart bandage with sensors for blood clots and infection. The picked data is transmitted to the clinic automatically. It comprises of reusable electronics for wireless monitoring of $\mathrm{pH}$ and infection. Chronic status is facilitated by real time monitoring of the bacterial infection. The bandage may be useful for real time monitoring over a long period of time [54]. Konwar [55] et al reported a low toxic, biocompatible, and low cost bandage of cotton patch coated with chitogen, a non-sticking material. The cotton loaded with drugs serves as $\mathrm{pH}$ sensitive delivery mechanism. Change in the $\mathrm{pH}$ due to bacterial infection initiates the drug release from the cotton patch. Lin [56] et al designed a temperature sensitive transparent, soft, durable, and flexible hydrogel bandage. Amount of drugs diffusion depends upon the temperature variation and the bandage is shown in Fig. 3 [56]. 


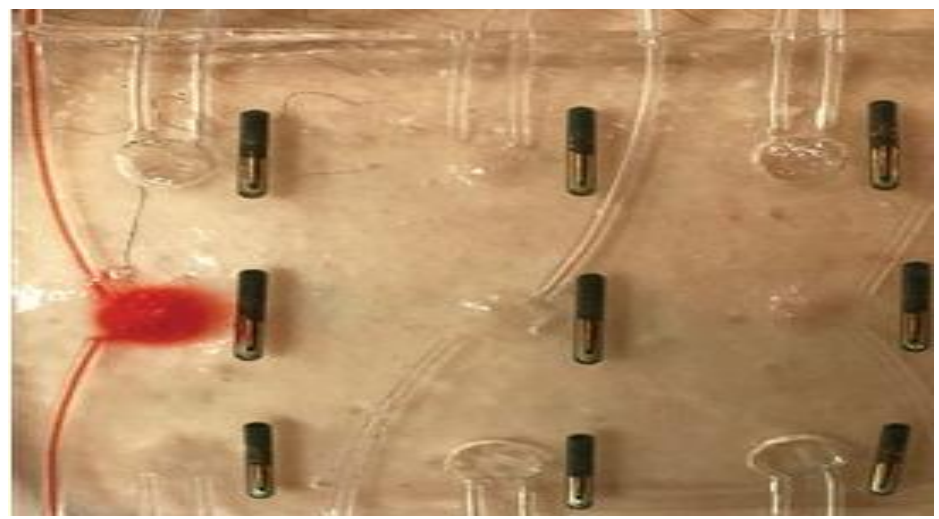

Fig. 3 A smart bandage designed by MIT researchers consisting of drug reservoirs, temperature sensors fabricated in a hydrogel matrix [56].

Swisher [57] et al reported a smart bandage detecting bed sores working on the principle of impedance spectroscopy. It consists of dozens of electrodes onto a thin flexible film for measuring skin impedance changes [57]. The smart bandage shown in Fig. 4 has been developed by Mostafalu [58] et al. It contains flexible $\mathrm{pH}$ sensors and a heater that triggers thermo-responsive drug carriers containing antibiotics. The thermoresponsive carriers are implanted in a layer of hydrogel around the $\mathrm{pH}$ sensors on top of the flexible heaters. It is equipped with an electronic module that records the sensor signals and powers the heaters.

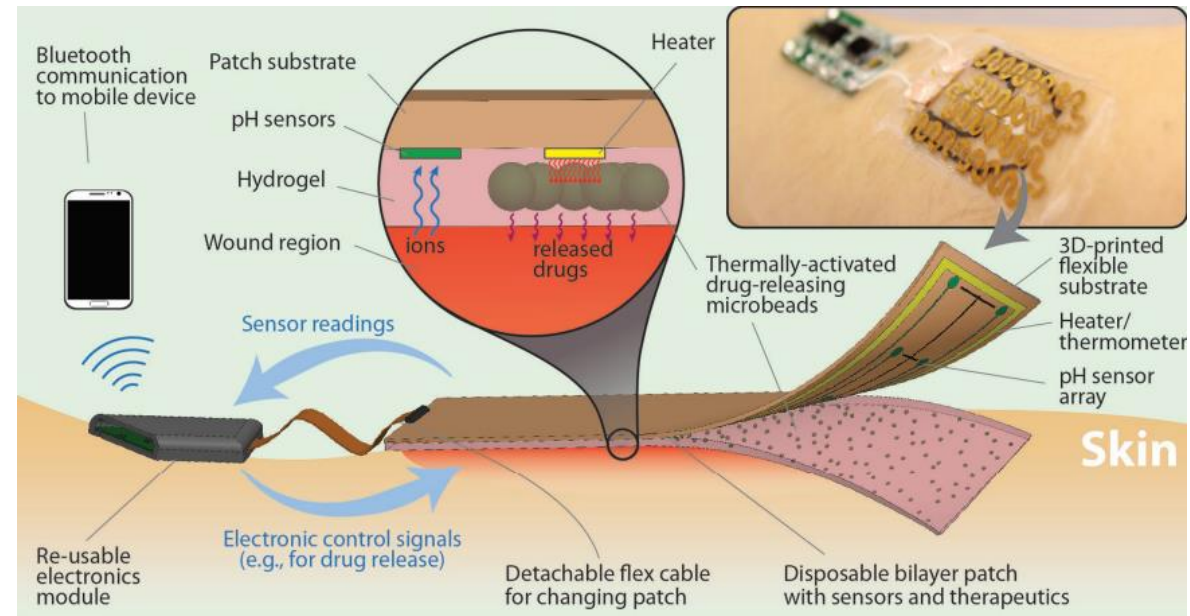

Fig. 4 A wireless smart bandage with automated drug delivery system [58].

The literature review on the design and development of smart bandages shows that preliminary work has been carried out at different laboratories in the world, but, there is a scope of dedicated research for developing ergonomic herbal bandages with special emphasis to low cost of the final product for making it more useful for the masses at large. Towards this end, simulation of the flow of drugs through the bandage within a specific time is necessary to speed up the tissue regeneration during the four stages of healing.

\section{Proposed Structure and Methodology}

Several advancements in smart bandage development were discussed in the last section. It was concluded that use of herbal drugs, dc current flow, heating, $\mathrm{pH}$ stabilization, moisture control, controlled amount of drug flow, and maintenance of $\mathrm{O}_{2}$ level are the main factors for fast healing of the damaged tissues. Towards this end, a smart bandage structure is proposed in this paper and investigations have been carried out to finalize the design of the bandage. The proposed structure is presented in the following subsection and the investigations carried out are discussed in Subsection 2.2.

\subsection{Proposed Structure of the Bandage}

Literature review on smart bandage design reveals that a blend of ayurvedic science and microfluidic technology may help in healing the wound effectively, based on this, a proposed structure of the smart bandage is shown in Fig. 5. The drugs are supplied to the wounded area through microfluidic channels. The flow rate is governed by laminar flow, capillary action, and diffusion phenomena. Because of the use of herbal ingredients as drug assures effective healing without any side effects to avoid formation of chronic wounds. 


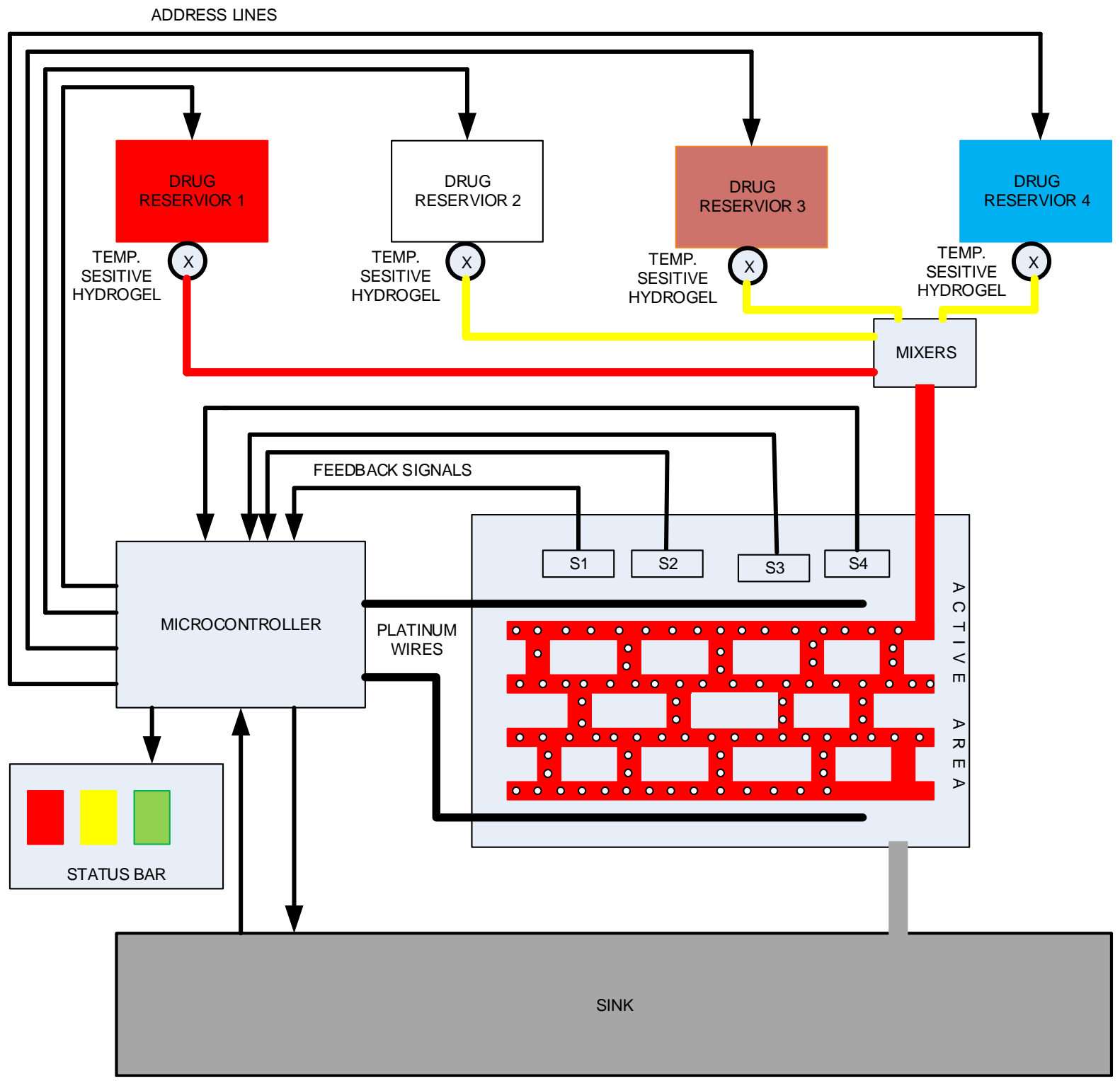

Fig. 5 Block Diagram of microfluidic bandage system

The bandage structure consists of five main blocks i.e. active area, status bar, drug chambers, sink, mixers, and a microcontroller for governing each activity depending upon the inputs from the wound. Active area of bandage is the most important sensitive part composed of porous material (Fig. 9) and laid with microfluidic channels for supplying of drugs to the wound. Reservoirs supply controlled amount of drug to the wound through the mixers. The status of drug reservoirs being full or empty is displayed on the status bar. The flow of drugs from chambers to the active area is controlled by heat stimuli. The efficiency of the mixing depends upon the dc voltage applied to the electrodes of electroosmotic mixer (Fig. 7). Three mixers are used subsequently to mix the drugs from the four reservoirs (Fig. 8). Change in $\mathrm{pH}$, oxygen, and moisture level measured by three sensors placed in active area, is displayed on the status bar. Variation in these parameters controls the drug flow from the reservoirs. Microcontroller is the main part of the bandage that controls the overall working of the bandage as per the information received from the sensors. The bandage has the facility of discarding the residual drug in the sink. Two platinum wires placed in the active area apply dc voltage of $3.3 \mathrm{~V}$. The heat produced due to dc current helps in healing of the wound. The bandage is composed of reusable (electronic components) and disposal parts to make it cost-effective. The active area laid with microfluidic channels consists of porous material placed in direct contact with the wound to supply the drugs effectively.

\subsection{Investigations}

In this research paper, three studies are carried Fabrication of drug chambers and microlfuidic channels, design and simulation of mixers, and simulation of active area of bandage.

Fabrication of drug chambers and microfluidic channels 
There are four drug chambers filled with neem oil and honey automatically controlled by microcontroller. Drug chamber are made of heat shrinkable tubes wrapped with a nichrome wire. Microfluidic channels are attached to the chambers (Fig. 6). Fluid control block consists of dc power, nichrome wire as heater, and a small MOSFET. On passing current through the nichrome wires, the heat produced shrinks the tubes and drug flows out of the drug chambers. Experiments were conducted with different dimensions of microfluidic channels for finalizing the optimal diameter of the channels.

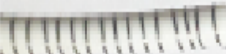

Fig. 6 Nichrome wire wrapped on heat shrink tube acts as current controllable reservoir.

The microfluidic channels are made of Polydimethylsiloxane by mixing elastomer and curing agent in the ration $1: 10$. The mixing ratio is carefully monitored as a slight change may deteriorate the rigidity or elasticity of the channels. The mixture is continuously stirred to avoid formation of bubbles and finally put inside a mould for developing microfluidic channels. The PDMS along with the mould was put inside a vacuum chamber for 24 hours to stabilize the structure. The mould was developed using a high precision CNC machine. In the present investigations, the plane engraved with microfluidic channels was sealed with a glass substrate. Investigations are carried out to study the flow of drugs with in the microfluidic channels of different dimensions for optimizing the flow rate of drugs as a result of applying heat stimuli to the reservoirs.

Design, fabrication, and simulation of mixers

The mixing block consisting of three mixers is shown in the Fig. 8. Fig. 7 shows the image of one of the mixers simulated. It consists of four electrodes $E_{0}, E_{1}, E_{2}, E_{3}$. The mixers were made inside PDMS using moulds of different dimensions. Investigations were also carried out using COMSOL Multiphysics based simulations to finalize the optimal dimensions of the mixers for proper flow and mixing of the drugs. Mixing of the drugs is essential for fast healing of the wound and protection against bacterial infection.

The microfluidic flow is simulated by using Navier stokes equations for incompressible flow

$$
\rho \frac{\partial u}{\partial t}-\nabla . \eta\left(\nabla u+(\nabla u)^{T}\right)+\rho u . \nabla u+\nabla p=0
$$

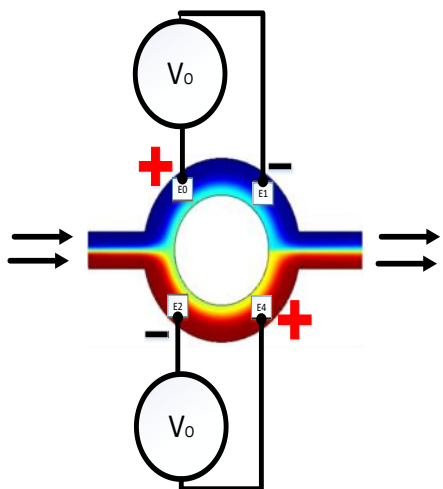

Fig. 7 Geometrical structure of mixer.

$\nabla . u=0$

Here $\eta$ denotes the dynamic viscosity, $u$ is the velocity $(\mathrm{m} / \mathrm{s}), \rho$ equals the fluid density $\left(\mathrm{kg} / \mathrm{m}^{3}\right)$, and $p$ refers to the pressure $(\mathrm{Pa})$. The boundary condition was taken as

$$
n \cdot\left\lfloor-p I+\eta\left(\nabla u+(\nabla u)^{T}\right)\right\rfloor=0
$$

It is assumed that solid surface attain a charge on coming in contact with electrolytes resulting in a charged solution close to the liquid-solid interface known as electric double layer. The electric field applied generates electro-osmotic flow and displaces the charged liquid imposing a force on the positively charged solution close to the wall surface to flow in the direction of the electric field. The velocity gradients perpendicular to the wall give rise to viscous transport in this direction. In the absence of other forces, the velocity profile eventually becomes almost uniform in the cross section perpendicular to the wall. The double layer is simulated using Helmholtz-Smoluchowski relation between the electro-osmotic velocity and the tangential component of the applied electric field.

$u=\frac{\varepsilon_{r} \zeta_{0}}{\eta} \nabla_{T} V$

where $\varepsilon_{w}=\varepsilon_{0} \varepsilon_{r}$ is fluid electric permittivity, $\zeta_{0}$ channel wall potential, and $\mathrm{V}$ the applied potential. This equation is valid at all boundaries except inlet and the outlet. Assuming that there are no concentration gradients in the ionic current, the current balance can be written as

$$
\nabla \cdot(-\sigma \nabla V \cdot n)=0
$$

where $\sigma$ denotes conductivity and the expression within parentheses represents the current density. The electric potentials on the electrodes are varied from $0 \mathrm{~V}$ to $3 \mathrm{~V}$. Assuming the remaining boundaries as insulated, insulation boundary condition may be written as

$$
-\sigma \nabla V \cdot n=0
$$


Initial concentrations at the inlets is fixed as $\mathrm{C} 1$ and $\mathrm{C} 2$, respectively. The convection-diffusion equation describing the concentration $c$ inside the mixer can be written as

$\frac{\partial c}{\partial t}+\nabla \cdot(-D \nabla c)=R-u \cdot \nabla c$

where $\mathrm{D}$ represents the diffusion coefficient, $\mathrm{R}$ the reaction rate, and $\mathrm{u}$ represents the flow velocity. Here $\mathrm{R}$ can be taken as 0 as there is no chemical reaction inside the mixer. Because the mixer is connected to microfluidic channels and the liquid flow through them is governed by the

$$
\rho\left[\partial_{t}+(v . \nabla) v\right]=-\nabla p+\eta \nabla^{2} v+\beta \eta \nabla(\nabla . v)+\rho g+\rho_{e l} E
$$

where $v$ is the fluid velocity, $p$ the fluid pressure, $\rho$ the fluid density, $\eta$ the fluid dynamic viscosity, and $g$ is the acceleration due to gravity. The term $\rho\left[\partial_{t} v+(v . \nabla) v\right]$ represents the inertial forces, $\nabla p$ the pressure forces, the term $\eta \nabla^{2} v+\beta \eta \nabla(\nabla . v)$ the viscous forces, $\rho g$ the gravitational forces, and $\rho_{e l} E$ represents the external forces acting on the fluid.

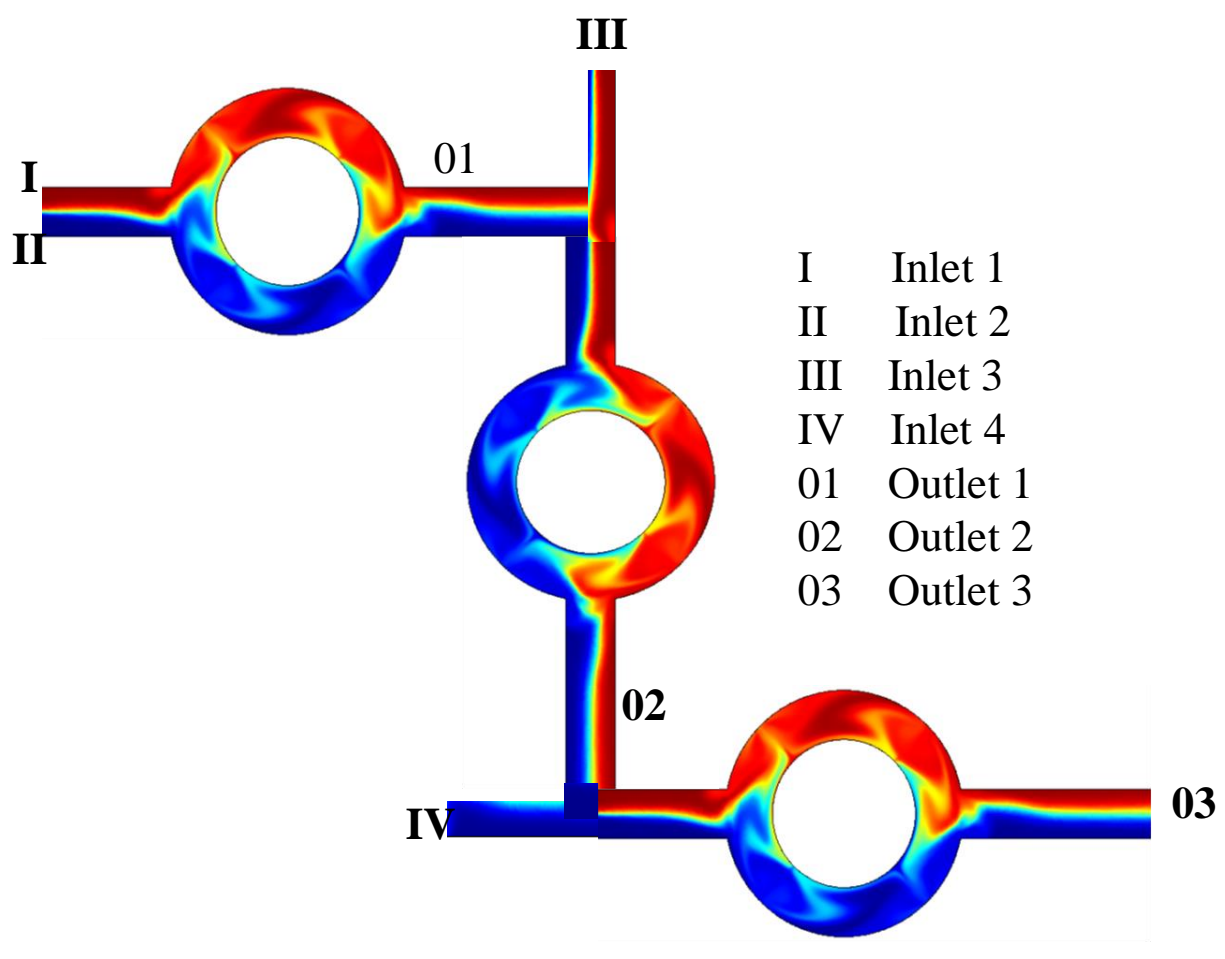

Fig. 8 A set of three mixers interconnected with each other to mix the drugs from four different drug reserviors. For laminar flow, the equations can be modified as stationary studies laminar flow

$\rho \frac{\partial u}{\partial t}+\rho(u . \nabla) u=\nabla \cdot\left[-\rho I+\mu\left(\nabla u+(\nabla u)^{T}\right)\right]+F$

Design and simulation of Active area of bandage

Active area involving microfluidic channels and porous material is shown in Fig. 9. The flow of drugs through the porous material is simulated by the physics of capillary and diffusivity based mechanisms. The detail of these mechanism is given as follows.

Effect of capillary action on the flow of drugs

The material is assumed to be made up of small pores obeying the following equation involving the term $F_{s t}$ as the force due to surface tension.

$\rho \frac{\partial u}{\partial t}+\rho(u \cdot \nabla) u=\nabla \cdot\left[-p I+\mu\left(\nabla u+(\nabla u)^{T}\right)\right]+F_{s t}+\rho g$

where $F_{s t}=\sigma \delta K n, n$ the interface normal, $\sigma$ the surface tension coefficient, $K=-\nabla . n$ the curvature, and $\delta$ is the Dirac delta function that is nonzero only at the fluid interface. For contact angle $(\theta)$, the liquid air interface 
force is given as $F_{\theta}=\sigma \delta\left(n_{\text {wall }} . n-\cos \theta_{w}\right) n$. In case of phase field interface, the surface tension force be written as $F_{s t}=\lambda\left[-\nabla^{2} \phi+\frac{\phi\left(\phi^{2}-1\right)}{\varepsilon^{2}}\right] \Delta \phi$ where $\phi$ is the phase field parameter.

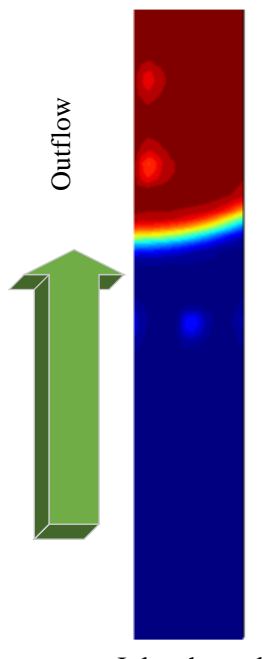

Inlet channel

(a)

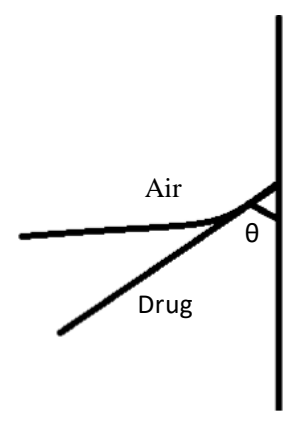

(b)

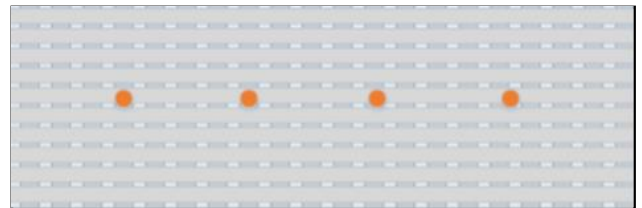

(c)

Fig. 9 Microfluidic flow through active area a) meniscus formation b) angle formation at meniscus c) a portion of active area of bandage

Effect of diffusion on the flow of drugs

Flow of drugs through the porous structure can also be modelled using diffusion based equations as

$\frac{\partial c}{\partial t}+\nabla \cdot(-D \nabla c)=0$

where D represents the diffusion coefficient. For inlet concentration $\mathrm{c}_{1}$, the outlet boundary condition is set as $(-D \nabla c) . n=k_{m}\left(c-c_{1}\right)$ where $\mathrm{k}_{\mathrm{m}}$ is the mass transfer coefficient [59].

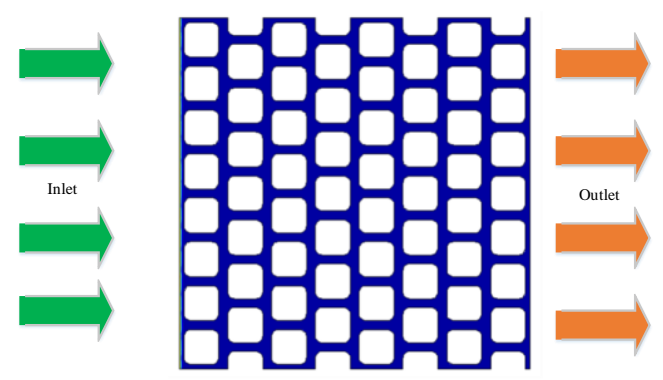

Fig. 10 Diffusivity structural model

\section{Results}

The proposed smart bandage was fabricated and investigated in parts as prototypes. As already discussed in Section 2.1, the different parts of the bandage consist of drug reservoirs, microfluidic channels, mixers, and active area. The results of investigations carried out with these sections of the bandage are described as follows.

\subsection{Fabrication of reservoirs and microfluidic channels}

Investigations with different materials and sizes for the fabrication of drug reservoirs were carried to optimize the dimension of drug chambers. In investigations show that transparent heat shrink tubes of radius $2 \mathrm{~mm}$ having length of about $4 \mathrm{~cm}$ are sufficient for holding the drugs needed for the duration of the expected usage time of the bandage, i.e. about one week. A dc voltage of $3.5 \mathrm{~V}$ is sufficient to push the drug out of the reservoir because of the heat generated in the nichrome wire having a resistance of $15 \mathrm{ohm}$. It was investigated that change in voltages controls the drug distribution. Automatic control of the heat is necessary to avoid the bursting of the microfluidic channels. One instance of the flow of drug inside a channel of radius 500 microns and length $4 \mathrm{~cm}$ is shown in Fig. 11. It was noticed that the drug requires $40 \mathrm{~ms}$ to reach active area of the bandage. 


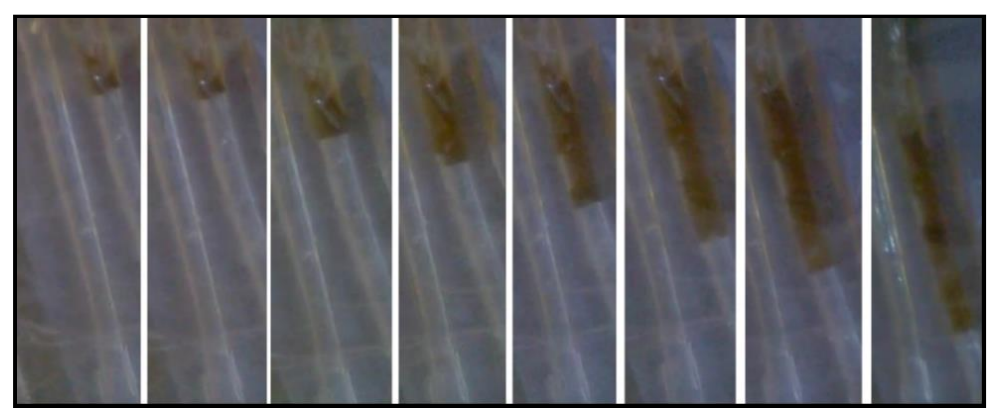

(a)

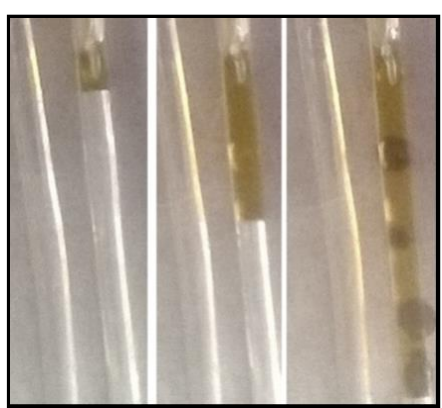

(b)

Fig. 11 Flow of neem oil across a microfluidic channels of length $4 \mathrm{~cm} 40 \mathrm{~ms}$.

\subsection{Simulation and fabrication of mixers}

Simulation based investigations were carried out for finalizing the size and shape of the mixer. It was seen that a mixer having inner radius as 150 microns and outer radius as 250 microns was sufficient to mix the incoming drugs via inlets of 50 microns diameter microfluidic channels. The experiment was carried out with different voltages ranging from $0 \mathrm{~V}$ to $3 \mathrm{~V}$ applied across the electrodes of mixer shown in Fig. 7. The results of the simulations are shown in Fig. 12. The concentration is represented in colors. Minimum concentration is shown by dark blue and maximum by red. For $0 \mathrm{~V}$, no change in the concentration was observed. Increase in potential provides better mixing as displayed by changing color. It may be concluded that a potential of $3 \mathrm{~V}$ is enough for better mixing. Although we could fabricate mixers in the dimensions of about 1200 microns, but, investigations of the mixers with the dimensions around 100 microns are not complete because of some complications involved for placement of electrodes and coupling of microfluidic channels with the mixers.

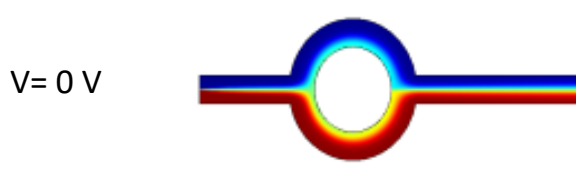

(a)

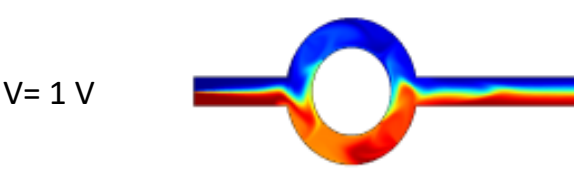

(c)

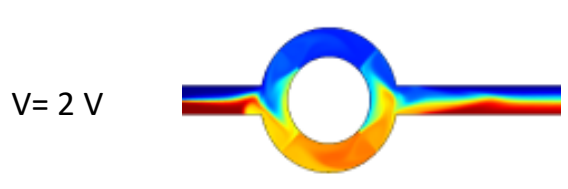

(e)

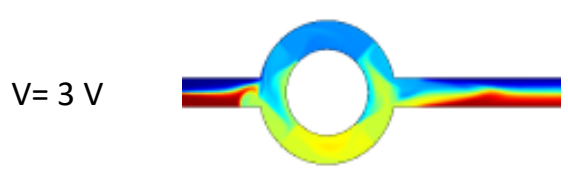

(g)

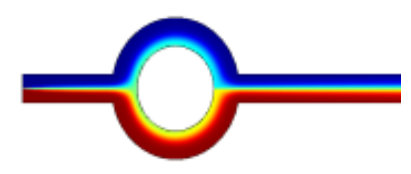

(b)

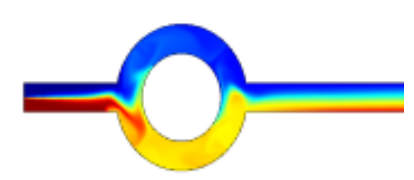

(d)

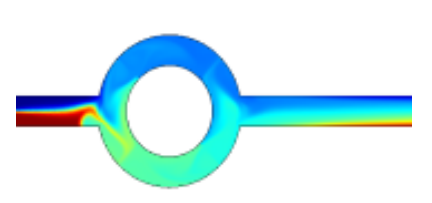

(f)

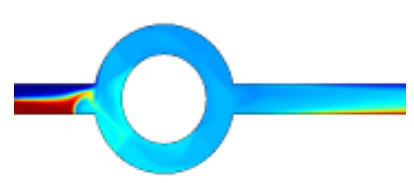

(h)

At time $2 \mathrm{~s}$ At time $10 \mathrm{~s}$

Fig. 12 Effect of varying potential on mixing of drugs at different instances of time. 


\subsection{Investigations of the active area of the bandage}

The drug flow through active area of bandage was investigated using capillary physics and diffusion phenomenon. Experimentation with different dimensions of capillaries ranging from 50 microns to 200 microns were carried as the diameters of most of the porous materials useful for bandage fall in this range. The results of simulation of the flow of drug through the capillary channel of width 150 microns and height $2 \mathrm{~cm}$ for the assumed simplified shape of the porous fibre shown in Fig. 9 are given in Table I and plotted in Fig. 13. The investigations have been carried out using only vertical capillaries as the flow through horizontal capillaries will be relatively faster because of the lesser impact of gravitation. The orientation of channels can be horizontal, or vertical as per the wound. As observed from the table, the displacement of drug increases with time and it takes only $1 \mathrm{~s}$ to reach a distance of $11.14 \mathrm{~mm}$.

Table 1 Displacement of neem oil against the action of gravity using capillary physics

\begin{tabular}{lllllllllllll}
\hline Time (s) & 0 & 0.1 & 0.2 & 0.3 & 0.4 & 0.5 & 0.6 & 0.7 & 0.8 & 0.9 & 1 & \\
& & & & & & & & & & & \\
Displacement (mm) & 0 & 2.91 & 4.31 & 5.44 & 6.43 & 7.34 & 8.18 & 8.96 & 9.71 & 10.43 & 11.14 \\
\hline
\end{tabular}

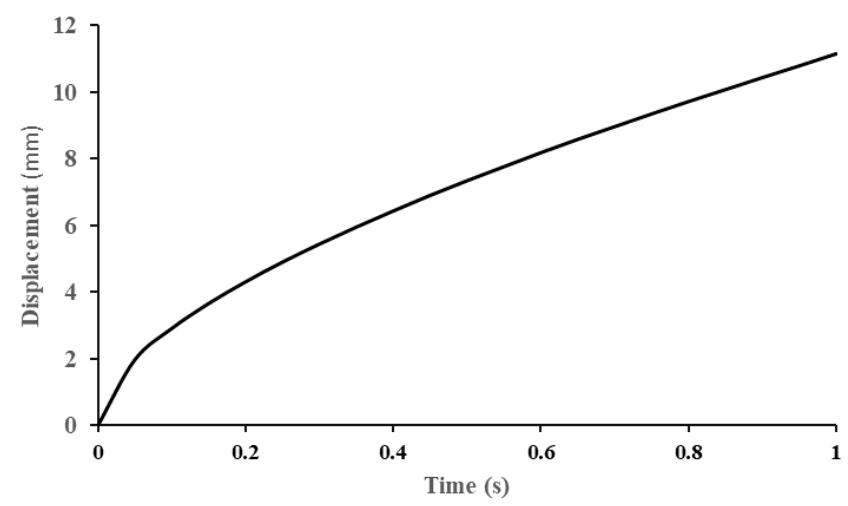

Fig. 13 Neem oil reached to the height of $11.14 \mathrm{~mm}$ in 1 second.

The results of simulation of the spread of drug diffusion across the porous material of size 800 microns' x 800 microns (Fig. 10) are shown in Fig. 14. From the figures showing the flow at different instants of time, it may be noted that the drug takes about $60 \mathrm{~ms}$ to reach a distance of 250 micro meter. The investigations using capillary and diffusion based studies show that capillary action dominates the flow due to diffusion.

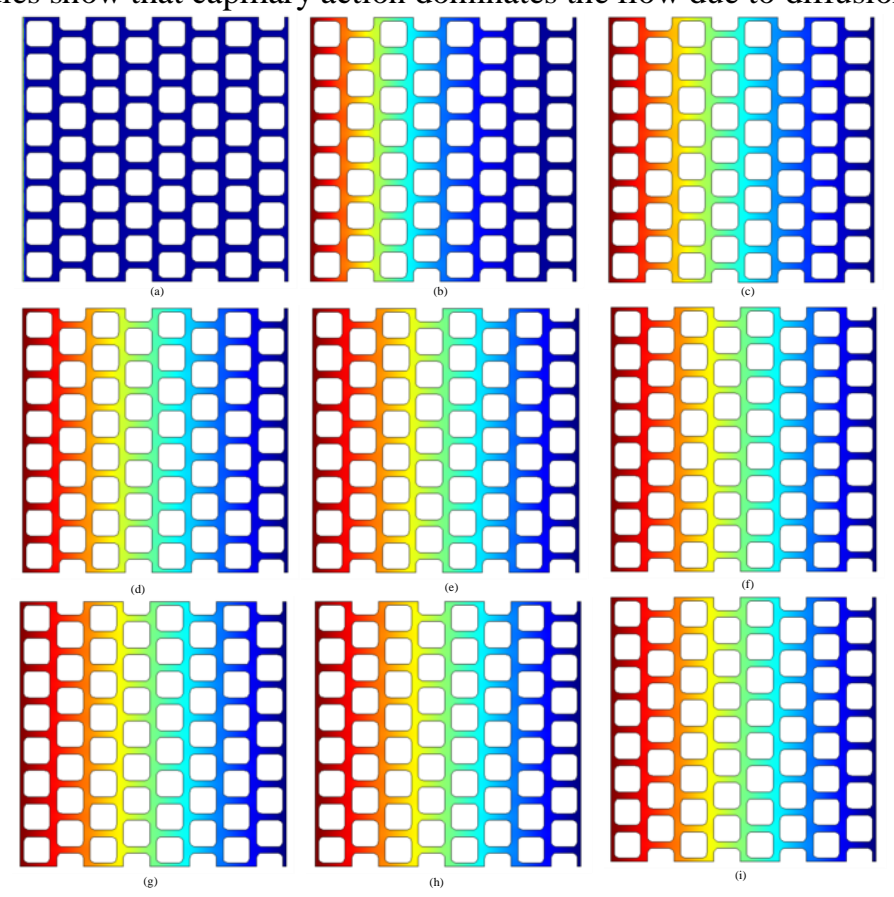

Fig. 14 Flow of drug through the porous material placed in active area of the bandage at time instances $0 \mathrm{~ms}, 10$ $\mathrm{ms}, 20 \mathrm{~ms}, 30 \mathrm{~ms}, 40 \mathrm{~ms}, 50 \mathrm{~ms}, 60 \mathrm{~ms}, 70$, and $80 \mathrm{~ms}$ due to diffusion phenomenon. 


\section{Conclusion}

Microfluidics is finding its application in flexible electronics for developing artificial human organs. In the present research a prototype smart bandage has been simulated and tested for its applications in speedy wound healing. It is capable of sensing the status of the wound using $\mathrm{pH}$ and moisture. The constituents of the proposed smart bandage are microfluidic channels, electroosmotic mixers, heat stimuli based drug reservoirs, dc current mechanism for fast healing, porous material, and small sinks for collecting used drugs. Drug reservoirs of different dimensions were fabricated and investigated for optimization of dimensions. The efficiency of the mixing depends upon the dc voltage applied to the electrodes of electroosmotic mixer. Simulation based investigations carried out for finalizing the size and shape of the mixers show that a mixer having inner radius as 150 microns and outer radius as 250 microns is sufficient for proper distribution of the drugs. The drug flow through active area of bandage was investigated using capillary physics for channels with dimensions ranging from 50 microns to 200 microns. Diffusivity effect on the porous material of dimension 800 microns was carried out and it can be concluded that capillary action for drug supply to effective area is relatively more effective as compared to diffusion phenomenon. Hence, a smart bandage with provisions of uniform drug distribution, automatic control, on board $\mathrm{pH}$ measurements, and dc current based healing mechanism is possible to be incorporated with for fast wound healing with in economical budget. The smart bandage may be a boon to the soldiers in battle fields, human settlements in remote areas, and astronauts in space where finding a doctor is difficult in the hour of need.

\section{References}

1. Tang DP, Chen LJ. A Review of the Evolution of Research on Information Technology Acceptance model. In: International conference on business management and electronic information 2011; 5: 588591.

2. Karimi M, Bahrami S, Mirshekari H, Seyed MMB, Amrila BN et al. Microfluidic systems for stem cellbased neural tissue engineering. Lab on a Chip 2016; 16(14): 2551-2571. DOI.10.1039/c6lc00489j

3. Liu Y, Jiang X. Why microfluidics? Merits and trends in chemical synthesis. Lab on a Chip 2017;17(23):3960-78. Available from: DOI.10.1039/c7lc00627f.

4. Peplow M. Organci Synthesis: The robo-chemist. Nature 2014; 512: 20-22.

5. Iqbal MH, Aydin A, Brunckhorst O, Dasgupta P, and Ahmed K. A review of wearable technology in medicine. Journal of the Royal Society of Medicine 2016; 109(10): 372-380.

6. Udovicic G, Topic A, and Russo M. Wearable technologies for smart environments: A review with emphasis on BCI. 24th International Conference on Software, Telecommunications and Computer Networks (SoftCOM) 2016.

7. Li JD, Wang SJ, Li KSM, and Ho TY. Test and diagnosis of paper-based microfluidic biochips 2016 IEEE 34th VLSI Test Symposium (VTS).

8. Liu S, Su W, and Ding X. A Review on Microfluidic Paper-Based Analytical Devices for Glucose Detection. Sensors 2016; 16(12): 2086.

9. Streets AM, Huang Y. Chip in a lab: Microfluidics for next generation life science research. Biomicrofluidics 2013; 7(1): 2013.

10. Potkay JA. The promise of microfluidic artificial lungs. Lab Chip 2014; 14(21): 4122-4138.

11. Thompson AJ, Marks LH, Goudie MJ, Rojas-Pena A, Handa H, and Potkay JA. A small-scale, rolledmembrane microfluidic artificial lung designed towards future large area manufacturing. Biomicrofluidics 2017; 11(2).

12. Damiati S, Kompella UB, Damiati SA, and Kodzius R. Microfluidic Devices for Drug Delivery Systems and Drug Screening. Genes (Basel). Genes 2018; 9(2):103. DOI.10.3390/genes9020103.

13. Dai J, Hamon M, and Jambovane S. Microfluidics for Antibiotic Susceptibility and Toxicity Testing. Bioengineering 2016; 3(4): 25. DOI.0.3390/bioengineering3040025.

14. Wang Z, Gao H, Zhang Y, Liu G, Niu G, and Chen X. Functional ferritin nanoparticles for biomedical applications. Frontiers of Chemical Science and Engineering. Springer Nature 2017;11(4): 633-46.

15. Leonard EE, Xiaobou Y, Zheng D, and Silverstein MD. The Prevalence and Pattern of Complementary and Alternative Medicine Use in Individuals with Diabetes, Diabetes Care 2002; 25(4): 324-329.

16. Chauhan A, Semwal DK, Mishra SP, and Semwal RB. Ayurvedic research and methodology: Present status and future strategies. An International Quarterly Journal of Research in Ayurveda 2015; 36(4): 364. DOI.10.4103/0974-8520.190699

17. Biswas T. Chapter 10. A Review on Indian Medicinal Plants for Wound Management. The Basic Needs to Achieve Wound Healing 2011;130-44.

18. Singh A, Singh A, Narayan G, Singh T, and Shukla V. Effect of Neem oil and Haridra on non-healing wounds. An International Quarterly Journal of Research in Ayurveda 2014; 35(4): 398-403. 
19. Kumar D, Shukla UK. Effect of turmeric (Curcuma longa) powder in diet on growth performance of broiler chicks. The Asian Journal of Animal Science. Hind Agri Horticultural Society; 2017 ;12(1):81-4.

20. Molan PC. Why honey is effective as a medicine. 1. Its use in modern medicine, Bee World 1999; 80(2), 80-92.

21. Zohdi RM, Mukhtar SM, Said S, Azmi NAM, and Ali AA. A comparative study of the wound healing properties of Gelam honey and silver sulfadiazine in diabetic rats. IEEE Conference on Biomedical Engineering and Sciences (IECBES) 2014.

22. Alhashim M, Lombardo J. Mechanism of Action of Topical Garlic on Wound Healing. Dermatologic Surgery 2017.

23. Ejaz S, Chekarova I, Cho JW, Lee SY, Ashraf S, and Lim CW. Effect of aged garlic extract on wound healing: A new frontier in wound management. Drug and Chemical Toxicology 2009; 32(3): 191-203.

24. H M, G El, Aboshanab KM, Aboulwafa MM, and Hassouna NA. Antivirulence and wound healing effects of royal jelly and garlic extract for the control of MRSA skin infections. Wound Medicine 2016; $13: 18-27$.

25. Fares AE, Khaled D, Khalaf M, Elsayed A, and Ghazal R. Garlic extract and phonophoresis in wound healing: Histological and immunohistochemical study. Egyptian Journal of Histology 2017; 40(3): 31527.

26. Rahati S, Eshraghian M, Ebrahimi A, and Pishva H. Effect of spinach aqueous extract on wound healing in experimental model diabetic rats with streptozotocin. Journal of the Science of Food and Agriculture 2015; 96(7): 2337-43.

27. Afra R, Seied MM. Spinach: An important green leafy vegetable and medicinal herb. The $2^{\text {nd }}$ International Conference on Medicinal Plants, Orgainc farming, Natural and Pharmaceutical Ingredients 2019.

28. Singh A, Singh A, Narayan G, Singh T, and Shukla V. Effect of Neem oil and Haridra on non-healing wounds. An International Quarterly Journal of Research in Ayurveda 2014; 35(4): 398-403.

29. Halim E, and Mukhopadhyay AK. Effect of Ocimum Sanctum (TULSI) and Vitamin E on Biochemical Parameters and Retinopathy in Streptozotocin Induced Diabetic Rats. Indian Journal of Clinical Biochemistry 2006; 21(2): 181-188.

30. Cohen M. Tulsi - Ocimum sanctum: A herb for all reasons. Journal of Ayurveda and Integrative Medicine 2014; 5(4): 251-259.

31. Nevin KG, and Rajamohan T. Effect of Topical Application of Virgin Coconut Oil on Skin Components and Antioxidant Status during Dermal Wound Healing in Young Rats. Skin Pharmacology and Physiology 2010; 23(6): 290-297.

32. Nuutila K, Katayama S, Vuola J, and Kankuri E. Human Wound-Healing Research: Issues and Perspectives for Studies Using Wide-Scale Analytic Platforms. Advances in Wound Care (New Rochelle) 2014; 3(3): 264-271.

33. Papel Ed. Anatomy and Physiology of the Skin, Facial Plastic and Reconstructive Surgery 2009.

34. Obagi ZE. Part One Skin Anatomy and Physiology. Obagi Skin Health Restoration and Rejuvenation 2000; 1-14. DOI.10.1007/978-0-387-21801-4_1.

35. Rittié L. Cellular mechanisms of skin repair in humans and other mammals. Journal of Cell Communication and Signaling 2016; 10(2): 103-120. DOI.10.1007/s12079-016-0330-1.

36. Leonida MD, and Kumar I. Wound Healing and Skin Regeneration, Bionanomaterials for Skin Regeneration 2016. 17-25, 2016.

37. Sun Z, and Williams GM. Skin Wound Healing. In Situ Tissue Regeneration 2016; 345-368. DOI:10.1016/b978-0-12-802225-2.00018-0.

38. Sanon DA, Hart, and Tredget EE. Molecular and Cellular Biology of Wound Healing and Skin Regeneration. Skin Tissue Engineering and Regenerative Medicine 2016; 19-47.

39. Golberg A. Scarless Tissue Regeneration. Bioengineering in Wound Healing, Wound healing 2017.

40. Meaume S, and Humbert P. Skin Wound Healing Assessment, Agache's Measuring the Skin 2017; 503 512.

41. Sumithra M, and Amutha R. Functional Modification on Adhesive Bandage Using Natural Herbs. Journal of Textile Science and Engineering 2016.

42. Konwar A, Kandimalla R, Kalita S, and Chowdhury D. Approach to Fabricate a Compact Cotton Patch without Weaving: A Smart Bandage Material. ACS Sustainable Chemistry \& Engineering 2018; 6(5): 5806-5817.

43. Hardy ET, Wang YJ, Iyer S, Mannino RG, Sakurai Y et al. Interdigitated microelectronic bandage augments hemostasis and clot formation at low applied voltage in vitro and in vivo. Lab on a Chip, 2018.

44. Tamayol A, Najafabadi AH, Mostafalu P, Yetisen AK, Commotto M et al. Biodegradable elastic nanofibrous platforms with integrated flexible heaters for on-demand drug delivery. Scientific Reports 2017; 7(1): 9220. 
45. Amjadi M, Sheykhansari S, Nelson BJ, and Sitti M. Recent Advances in Wearable Transdermal Delivery Systems. Advanced Materials 2018; 30(7).

46. Pan N, Qin J, Pingping F, Li Z, and Song B. Color changing of smart fibrous materials for naked eye real-time monitoring of wound pH. Journals of Material Chemistry B 2019; 7(16): 2626-2633.

47. Sattar H, Bajwa IS, Amin R, Muhammad J, and Mushtaq MF. Smart wound hydration monitoring using biosensors and fuzzy Interference system. Wireless communication and Mobile computing 2019.

48. Fraunhofer-Gesellschaft. New bandages change color if infections arise. ScienceDaily 2010. <www.sciencedaily.com/releases/2010/11/101116093821.htm>.

49. Orcutt M. Smart Bandage Signals Infection by Turning Fluorescent. MIT Technology Review 2015.

50. https://www.technologyreview.com/s/544166/smart-bandagesignals-infection-by-turning-fluorescent/

51. Avnir Y, Barenholz Y. pH determination by pyranine: medium-related artifacts and their correction. Anal Biochem. 2005; 347(1):34-41. DOI: 10.1016/j.ab.2005.09.026.

52. Mostafalu P, Kiaee G, Giatsidis G, Khalilpour A, Nabavinia M et al. A Textile Dressing for Temporal and Dosage Controlled Drug Delivery. Advanced Functional Materials 2017; 27(41):1702399. DOI.10.1002/adfm.201702399.

53. Sayed P, Kaeppeli A, Siriwardena T, Darbre T, Perron K et al. Anti-Microbial Dendrimers against Multidrug-Resistant P. aeruginosa Enhance the Angiogenic Effect of Biological Burn-wound Bandages. Scientific Reports 2016; 6(1).

54. Farooqui MF, and Shamim A. Low Cost Inkjet Printed Smart Bandage for Wireless Monitoring of Chronic Wounds. Scientific Reports 2016; 6.

55. Farooqui MF, Shamim A. Inkjet printed wireless smart bandage. IEEE Middle East Conference on Antennas and Propagation (MECAP), Beirut, 2016. DOI: 10.1109/MECAP.2016.7790102.

56. Konwar A, Kandimalla R, Kalita S, and Chowdhury D. Approach To Fabricate a Compact Cotton Patch without Weaving: A Smart Bandage Material. ACS Sustainable Chemistry \& Engineering 2018; 6(5): 5806-5817.

57. Lin S, Yuk H, Zhang T, Parada GA, Koo H et al. Stretchable Hydrogel Electronics and Devices. Advanced Materials 2015; 28(22), 4497-4505. DOI:10.1002/adma.201504152.

58. Swisher SL, Lin MC, Liao A, Leeflang EJ, Khan Y et al. Impedance sensing device enables early detection of pressure ulcers in vivo. Nature Communications 2015; 6(1).

59. Mostafalu P, Tamayol A, Rahimi R, Ochoa M, Khalilpour A et al. Smart Bandage for Monitoring and Treatment of Chronic Wounds. 2018.

60. Bruus H. Governing Equations in Microfluidics. Microscale Acoustofluidics 2014; 1-28. DOI. 10.1039/9781849737067-00001. 